\title{
The Utilization of 5-Oxoproline, Ammonia and Glutamine by Rhizobium leguminosarum in Chemostat Culture
}

\author{
By HOWARD D. RATCLIFFE, ${ }^{1} \dagger$ JAN W. DROZD ${ }^{1 *}$ AND ALAN T. BULL ${ }^{2} \ddagger$ \\ ${ }^{1}$ Shell Research Limited, Sittingbourne Research Centre, Sittingbourne, Kent ME9 8AG, U.K. \\ ${ }^{2}$ Department of Applied Biology, University of Wales Institute of Science and Technology, \\ King Edward VII Avenue, Cardiff CF1 3NU, U.K.
}

(Received 28 June 1982; revised 22 November 1982)

\begin{abstract}
The growth in continuous culture of Rhizobium leguminosarum on the nitrogen sources glutamine, ammonia, or ammonia and 5-oxoproline (formed when aqueous glutamine solutions are autoclaved) is described. When growth was nitrogen-limited with autoclaved glutamine the specific rate of 5-oxoproline utilization was independent of dilution rate, whereas the specific rate of ammonia utilization increased with dilution rate. Although the culture was nitrogenlimited, excess nitrogen (as 5-oxoproline) was still present in the supernatant, especially at high dilution rates.

Values obtained for $Y_{\text {glucose }}^{\max }$ and $Y_{\text {oxygen }}^{\max }$ were 72.2 and $32.5 \mathrm{~g}$ dry wt bacteria (mol substrate) $)^{-1}$, respectively, under ammonia/5-oxoproline limitation and 95.7 and $30.2 \mathrm{~g}$ dry wt bacteria (mol substrate) $)^{-1}$, respectively, under glutamine limitation.
\end{abstract}

\section{INTRODUCTION}

L-Glutamine has often been used as a fixed nitrogen source for the growth of rhizobia (Wilcockson \& Werner, 1978, 1979) without a realization that, on heating (e.g. autoclaving) in aqueous media, glutamine cyclizes forming equimolar amounts of pyrollidone carboxylic acid (5-oxoproline) and ammonia (Archibald, 1945; Ratcliffe et al., 1978). The ammonia produced could complicate and invalidate studies on the regulation of nitrogen fixation if no allowance was made for the thermal instability of glutamine solutions.

Wilcockson \& Werner (1979) acknowledged that their earlier studies (Wilcockson \& Werner, 1978 ) with glutamine as a nitrogen source did not take into account the thermolabile nature of aqueous glutamine solutions but in many other studies (e.g. Bergersen et al., 1976) it is unclear as to whether the work is compromised because insufficient experimental details are given as to how the glutamine solutions were sterilized.

There have been a few reports of 5-oxoproline metabolism by prokaryotes (Maruyama \& Nomura, 1956; Kawai et al., 1965, 1966, 1969; Tosa \& Chibata, 1965; Van der Werf \& Meister, 1974, 1975). L-Glutamic acid was the first detectable product of 5-oxoproline metabolism in every case. It is thus unclear as to whether both ammonia and 5-oxoproline nitrogen will be utilized simultaneously if heat sterilized glutamine is used as the growth limiting nutrient in chemostat studies. In batch cultures of Rhizobium leguminosarum and Klebsiella pneumoniae ammonia was rapidly used as a nitrogen source but growth was slower with 5-oxoproline as nitrogen source (Ratcliffe et al., 1978).

When filter sterilized L-glutamine was used as sole nitrogen source in batch cultures of $R$. leguminosarum equimolar amounts of ammonia were excreted (Ratcliffe et al., 1978). Thus the use of glutamine as a nitrogen source could also lead to equivocal results if the production of

† Present address : Biotechnology Department, Kuwait Institute for Scientific Research, P.O. Box 24885, Safat, Kuwait.

$\ddagger$ Present address: Biological Laboratory, University of Kent at Canterbury, Canterbury, Kent CT2 7NJ, U.K. 
ammonia is not assessed. It is not known if extracellular ammonia is also produced from glutamine when $R$. leguminosarum is grown glutamine-limited in continuous culture. Bergersen et al. (1976) did not measure ammonia concentrations when they studied nitrogenase activity in glutamine limited cultures of cowpea rhizobia.

The aim of the present study was to investigate the kinetics of utilization of the nitrogen sources 5-oxoproline and ammonia, ammonia, and glutamine in $R$. leguminosarum when grown aerobically under nitrogen limitation with glucose as the primary carbon source (glutamine and 5-oxoproline may act as secondary carbon sources) in continuous culture. The growth of $R$. leguminosarum under carbon and oxygen limitations is described in an accompanying paper (Ratcliffe et al., 1983).

\section{METHODS}

Organism and growth conditions. Details of the organism, growth medium and chemostat are given in Ratcliffe et al. (1983). The glucose concentration in the medium was $10 \mathrm{~g} \mathrm{l}^{-1}$. Glutamine, autoclaved glutamine (i.e. equimolar concentration of ammonia and 5-oxoproline) or ammonium sulphate were added as required to give a final concentration of $10 \mathrm{mM}$.

Glucose, $\mathrm{MgSO}_{4} \cdot 7 \mathrm{H}_{2} \mathrm{O}$ and $\mathrm{CaCl}_{2} \cdot 2 \mathrm{H}_{2} \mathrm{O}$ were autoclaved separately: $\mathrm{FeSO}_{4} \cdot 7 \mathrm{H}_{2} \mathrm{O}$, the nitrogen sources (glutamine or ammonia) and vitamins were filter sterilized (Millipore, $0.45 \mu \mathrm{m}$ pore size) before aseptic addition to the cool medium (30 1).

Polysaccharide determination. To $10 \mathrm{ml}$ of culture supernatant $1 \mathrm{ml}$ of $10 \%(\mathrm{w} / \mathrm{v})$ aqueous $\mathrm{KCl}$ solution and $20 \mathrm{ml}$ isopropyl alcohol were added with stirring. The precipitated polysaccharide was removed by winding around a glass rod and weighed after drying $\left(98^{\circ} \mathrm{C}\right)$ to constant weight.

Other analyses. $\mathrm{O}_{2}$ uptake and $\mathrm{CO}_{2}$ evolution rates in the chemostat, the determination of substrate dependent $\mathrm{O}_{2}$ uptake rates, $\mathrm{C}, \mathrm{H}$ and $\mathrm{N}$ analysis, total carbon and glucose analyses were made according to the methods described by Ratcliffe et al. (1983). Glutamine and ammonia were determined by the methods previously described (Ratcliffe et al., 1978).

\section{RESULTS}

\section{Ammonia/5-oxoproline-limited growth of Rhizobium leguminosarum}

The results (Fig. 1) of a nitrogen-limited chemostat experiment with autoclaved glutamine as nitrogen source showed that the biomass concentration decreased with an increase in dilution rate. Free ammonia was not found in the supernatant at the tested dilution rates, indicating that $R$. leguminosarum had a very low $K_{\mathrm{s}}$ for ammonia. The $K_{\mathrm{m}}$ for 5-oxoproline dependent $\mathrm{O}_{2}$ uptake was approximately $0.4 \mathrm{mM}$, independent of dilution rate. More 5-oxoproline was utilized at low than at high dilution rates which implied that the specific rate of 5-oxoproline utilization was independent of the specific growth rate. Figure 2 shows that the specific rate of 5-oxoproline utilization ( $q_{5 \text {-oxoproline }}$ ) was independent of dilution rate but as expected $q_{\text {ammonia }}, q_{\mathrm{O}_{2}}$ and $q_{\text {glucose }}$ [mmol substrate consumed ( $\mathrm{g}$ dry $\mathrm{wt})^{-1} \mathrm{~h}^{-1}$ ] showed a linear increase with increase in dilution rate.

The culture was subjected to a total nitrogen balance (nitrogen 'in' as ammonia and oxoproline compared with nitrogen 'out' as 5-oxoproline and biomass). The results (Table 2) were calculated from percentage dry weight of $\mathrm{C}, \mathrm{H}$ and $\mathrm{N}$ in freeze-dried bacteria given in Table 1 . The apparent loss of supplied nitrogen may have been due to an error in the assay for $\mathrm{NH}_{4}^{+}$or 5oxoproline, or to the presence of nitrogen-containing extracellular metabolites. No extracellular polysaccharide was produced as assayed by viscosity. The carbon recovery values (Table 3 ) also indicate that under the given conditions $R$. leguminosarum did not excrete high concentration of carbon-containing metabolites, however, it is not possible to calculate if much of the carbon from 5-oxoproline was assimilated because the amounts involved are small relative to the total supernatant carbon concentrations.

Values for $Y^{\max }$ (yield corrected for maintenance substrate consumption) of 72.2 and $32.5 \mathrm{~g}$ dry wt bacteria (mol substrate) $)^{-1}$, were obtained for glucose and oxygen, respectively, from the data presented in Fig. 2 for $R$. leguminosarum cultures grown under ammonia/5-oxoproline (i.e. nitrogen) limitation. Maintenance requirements were 0.03 and $0.07 \mathrm{mmol}(\mathrm{g} \mathrm{dry} \mathrm{wt})^{-1} \mathrm{~h}^{-1}$ for glucose and oxygen, respectively. 


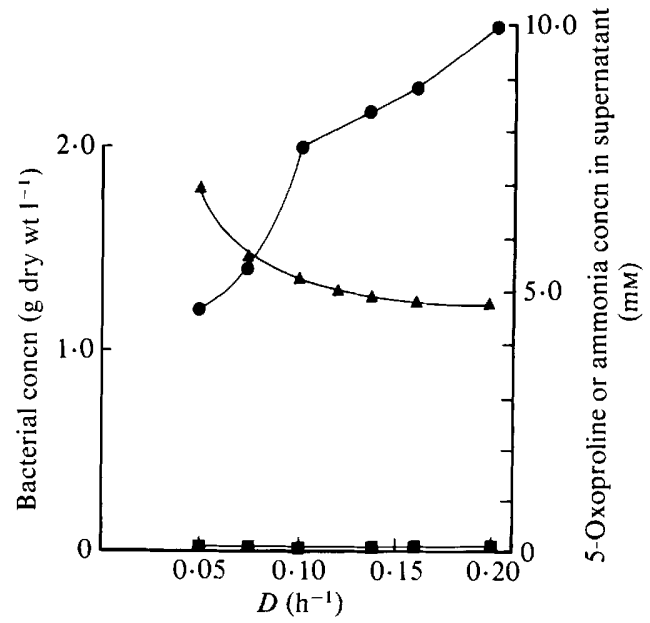

Fig. 1

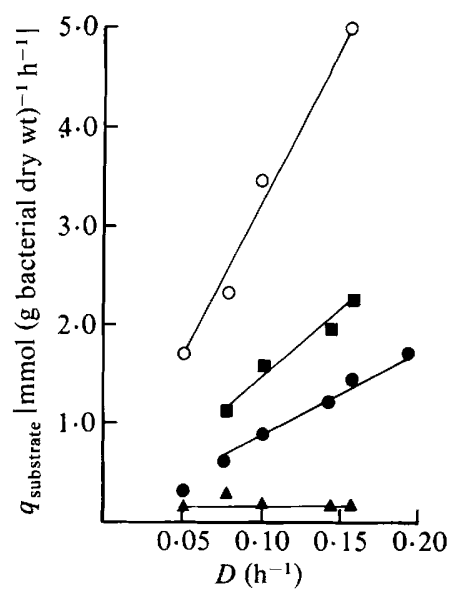

Fig. 2

Fig. 1. Relationship between biomass concentration and 5-oxoproline utilization in an ammonia/5oxoproline-limited chemostat culture of $R$. leguminosarum, $28^{\circ} \mathrm{C}, \mathrm{pH} 6 \cdot 8$. Bacteria were grown under nitrogen limitation at several dilution rates with glucose as the carbon source and autoclaved glutamine as the nitrogen source. $\boldsymbol{\Delta}$, Biomass (g dry wt $\mathbf{1}^{-1}$ ); $\boldsymbol{0}$, 5-oxoproline concentration (mM); $\mathbf{\square}$, ammonia concentration (mM).

Fig. 2. Relationship between $q_{\mathrm{NH}_{4}+}, q_{\text {5-oxoproline }}, q_{\mathrm{glucose}} ; q_{\mathrm{O}_{2}}$ and dilution rate for an ammonia/5-oxoproline-limited culture of $R$. leguminosarum. The nitrogen-limited culture was grown with autoclaved glutamine as the nitrogen source and glucose as the carbon source at $28^{\circ} \mathrm{C}, \mathrm{pH} 6.8$. Specific rates of ammonia utilization $(\boldsymbol{O})$, oxoproline utilization $(\boldsymbol{\Lambda})$, glucose utilization $(\boldsymbol{\square})$ and oxygen utilization $(O)$ are expressed as mmol (g dry wt $)^{-1} h^{-1}$.

Table 1. Elemental composition of $R$. leguminosarum grown under nitrogen limitation in chemostat culture

$\begin{gathered}\text { Date } \\ \left(\mathrm{h}^{-1}\right)\end{gathered}$
$\overbrace{\mathrm{C} \quad \mathrm{H}}^{\text {Composition }(\%)}$

(a) Glutamine limitation

$\begin{array}{llll}0 \cdot 05 & 46 \cdot 4 & 6.9 & 10 \cdot 5 \\ 0 \cdot 10 & 44.4 & 6.7 & 11 \cdot 6 \\ 0 \cdot 13 & 43.5 & 6.5 & 12 \cdot 2\end{array}$

(b) Ammonia limitation

$\begin{array}{llll}0.05 & 46.7 & 6.9 & 11.8 \\ 0.08 & 46.5 & 6.4 & 11.6 \\ 0 \cdot 10 & 46.1 & 6.7 & 11.3\end{array}$

(c) Ammonia/5-oxoproline limitation

$\begin{array}{llll}0 \cdot 05 & 45.3 & 6.9 & 10 \cdot 2 \\ 0.08 & 45 \cdot 2 & 6.8 & 11 \cdot 0 \\ 0 \cdot 10 & 44.2 & 6.7 & 10.8 \\ 0 \cdot 13 & 44.4 & 6.8 & 10.8 \\ 0 \cdot 16 & 44.7 & 6.9 & 11 \cdot 5 \\ 0.20 & 44.0 & 6.7 & 11.5\end{array}$

\section{Glutamine-limited growth of $R$. leguminosarum}

No extracellular ammonia was detected in glutamine-limited chemostat culture. Copious amounts of extracellular polysaccharide were found under glutamine (i.e. nitrogen) limitation. The specific rate of polysaccharide production increased from $0.018 \mathrm{~g}$ dry wt polysaccharide $(\mathrm{g}$ dry wt bacteria) $)^{-1} \mathrm{~h}^{-1}$ at $D=0.02 \mathrm{~h}^{-1}$ to 0.07 at $D=0.13 \mathrm{~h}^{-1}$. 
Table 2. Nitrogen balance in an ammonia/5-oxoproline-limited chemostat culture of

\section{R. leguminosarum}

Bacteria were grown under nitrogen limitation at $28^{\circ} \mathrm{C}, \mathrm{pH} 6.8$ with glucose as the carbon source. The $\mathrm{NH}_{4}^{+}$concentration in the supernatant was below the sensitivity of the analysis (approximately $50 \mu \mathrm{M}$ ). No allowance was made for possible $\mathrm{NH}_{4}^{+}$loss during autoclaving.

\begin{tabular}{|c|c|c|c|c|c|c|c|}
\hline$D\left(\mathrm{~h}^{-1}\right)$ & $\begin{array}{c}\text { Bacterial } \\
\text { dry wt } \\
\left(\mathrm{g} \mathrm{l}^{-1}\right)\end{array}$ & $\begin{array}{c}\text { Observed } N \\
\text { content of } \\
\text { dry bacteria } \\
(\%)\end{array}$ & $\begin{array}{l}\text { Total } \\
\text { bacterial } \\
\text { nitrogen } \\
\left(\mathrm{g} \mathrm{l}^{-1}\right)\end{array}$ & $\begin{array}{l}\text { 5-Oxoproline } \\
\text { concentration } \\
\text { in supernatant } \\
\left(\mathrm{g} \mathrm{l}^{-1}\right)\end{array}$ & $\begin{array}{c}\text { Total N } \\
\text { utilized } \\
\left(\mathrm{g} \mathrm{l}^{-1}\right)\end{array}$ & $\begin{array}{c}\text { Expected biomass } \\
\text { from } N \\
\text { content of cells } \\
\left(\mathrm{g}^{-1}\right)\end{array}$ & $\begin{array}{c}\text { Measured biomass } \\
\text { as \% of } \\
\text { expected biomass }\end{array}$ \\
\hline 0.05 & $1 \cdot 81$ & $10 \cdot 2$ & 0.185 & 0.595 & 0.221 & $2 \cdot 17$ & $83 \cdot 6$ \\
\hline 0.08 & 1.46 & $11 \cdot 0$ & 0.161 & 0.70 & 0.210 & 1.91 & 76.4 \\
\hline $0 \cdot 10$ & $1 \cdot 38$ & $10 \cdot 8$ & 0.149 & 0.99 & 0.173 & 1.60 & $86 \cdot 3$ \\
\hline 0.13 & $1 \cdot 27$ & $10 \cdot 8$ & 0.137 & 1.09 & $0 \cdot 158$ & $1 \cdot 46$ & $86 \cdot 8$ \\
\hline $0 \cdot 16$ & 1.22 & 11.5 & 0.140 & $1 \cdot 14$ & 0.162 & 1.41 & $86 \cdot 2$ \\
\hline $0 \cdot 20$ & $1 \cdot 26$ & $11 \cdot 5$ & 0.145 & $1 \cdot 39$ & $0 \cdot 142$ & $1 \cdot 23$ & $102 \cdot 0$ \\
\hline
\end{tabular}

Table 3. Supernatant carbon and unused carbon from glucose and 5-oxoproline in an ammonial 5-oxoproline-limited chemostat culture of $\mathrm{R}$. leguminosarum at $28^{\circ} \mathrm{C}, \mathrm{pH} 6.8$

\begin{tabular}{|c|c|c|c|c|c|}
\hline $\begin{array}{l}\text { Dilution } \\
\text { rate } \\
\left(\mathrm{h}^{-1}\right)\end{array}$ & $\begin{array}{l}\text { Carbon in } \\
\text { supernatant } \\
\text { as glucose } \\
\left(\mathrm{g} \mathrm{l}^{-1}\right)\end{array}$ & $\begin{array}{l}\text { Carbon in } \\
\text { supernatant as } \\
\text { 5-oxoproline } \\
\left(\mathrm{g} \mathrm{l}^{-1}\right)\end{array}$ & $\begin{array}{l}\text { Total observed } \\
\text { supernatant } \\
\text { carbon } \\
\left(\mathrm{g} \mathrm{I}^{-1}\right) \\
\text { (A) }\end{array}$ & $\begin{array}{l}\text { Total carbon } \\
\text { as 5-oxoproline } \\
\text { and glucose } \\
\left(\mathrm{g} \mathrm{l}^{-1}\right) \\
\text { (B) }\end{array}$ & $\begin{array}{c}\text { (B) as } \% \\
\text { of }(\mathrm{A})\end{array}$ \\
\hline 0.05 & $2 \cdot 02$ & 0.28 & $2 \cdot 53$ & $2 \cdot 30$ & $90 \cdot 8$ \\
\hline 0.08 & $2 \cdot 34$ & 0.33 & $2 \cdot 9$ & $2 \cdot 67$ & $92 \cdot 1$ \\
\hline $0 \cdot 10$ & $2 \cdot 43$ & 0.46 & $3 \cdot 15$ & 2.89 & $91 \cdot 7$ \\
\hline $0 \cdot 13$ & $2 \cdot 66$ & 0.51 & 3.33 & $3 \cdot 17$ & $95 \cdot 2$ \\
\hline $0 \cdot 16$ & 2.77 & 0.53 & $3 \cdot 35$ & $3 \cdot 30$ & 98.5 \\
\hline $0 \cdot 20$ & $2 \cdot 74$ & 0.61 & 3.65 & $3 \cdot 35$ & $91 \cdot 8$ \\
\hline
\end{tabular}

Rhizobium leguminosarum grown under glutamine (i.e. nitrogen) limitation gave $Y^{\text {max }}$ values of 95.7 and $30.2 \mathrm{~g}$ dry wt bacteria (mol substrate) ${ }^{-1}$ for glucose and oxygen, respectively, as determined from $q_{\text {substrate }}$ versus dilution rate data.

The $K_{\mathrm{m}}$ for glutamine-dependent oxygen uptake by washed culture samples of $R$. leguminosarum was $62.5 \mu \mathrm{M}$ and for glucose was 6 to $8 \mu \mathrm{M}$. Glutamine-dependent oxygen uptake activity indicated that the organisms had the potential to oxidize the carbon in glutamine, however, carbon recovery data were not accurate enough to determine if there was extensive incorporation of glutamine carbon in situ.

\section{Ammonia-limited growth of $R$. leguminosarum}

For an ammonia-limited chemostat elemental analysis (Table 1) indicated that the percentages of carbon, hydrogen and nitrogen in the organisms remained relatively constant with increase in dilution rate. Unlike the glutamine-limited culture very little extracellular polysaccharide was produced under these carbon sufficient conditions. There was no measurable ammonia present in the culture supernatant at any dilution rate.

\section{DISCUSSION}

When $R$. leguminosarum was grown under nitrogen limitation with autoclaved glutamine (ammonia plus 5-oxoproline), although the culture was nitrogen-limited, nitrogen (as 5-oxoproline) was present in the supernatant at high dilution rates. As the specific rate of 5-oxoproline utilization was independent of dilution rate the increase in the supernatant 5-oxoproline concentration with increase in dilution can be explained by the decrease in culture residence time as the dilution rate is increased; at low dilution rates there is a long residence time for the culture to 
assimilate the 5-oxoproline. As expected the specific rate of ammonia utilization increased with an increase in dilution rate. These results agree with batch culture studies which indicate that growth is much slower with 5-oxoproline than with ammonia as sole nitrogen source (Ratcliffe $e t$ al., 1978).

The results for nitrogen limitation with ammonia and 5-oxoproline are interesting with respect to the commonly used criteria for the definition of nutrient limitation in chemostat culture. Although nitrogen limited the culture cannot be said to conform to the usual criteria for nutrient limitation because of the low percentage of nitrogen as 5-oxoproline which is assimilated. It is the constant low value for $q_{5 \text {-oxoproline }}$ which limits the total amount of nitrogen assimilated to much less than near $100 \%$ (as it is under ammonia, or glutamine limitation). The low value for $q_{5 \text {-oxoproline }}$ probably reflects a very low but constant rate of transport or metabolism of this compound. Similar situations may occur frequently in natural environments where recalcitrant nitrogen containing compounds are present in addition to readily utilized compounds.

The low values of $Y_{\text {oxygen }}^{\max }$ for $R$. leguminosarum grown under nitrogen limitation with the nitrogen sources used, are similar to those found for carbon limitation (Ratcliffe et al., 1983). This is probably indicative of a high membrane permeability to protons (Ratcliffe et al., 1983), because respiration-induced proton translocation experiments and the cytochrome spectra (Ratcliffe et al., 1983) are indicative of three potential proton-translocating loops (Jones et al., 1977; Jones, 1978). There is a complication in measuring the glucose and oxygen yield values with glutamine and 5-oxoproline as nitrogen sources. Extra carbon and energy can be derived from these substrates, although the maximum amounts available are small in comparison with the amounts of glucose utilized. The carbon recovery data were not accurate enough to determine if extensive utilization occurred, but the organisms had the potential to oxidize the carbon in glutamine and 5-oxoproline. Also because they are more complex nitrogen sources than ammonia they will require less energy for incorporation into biomass, i.e. they will increase the $Y_{\mathrm{ATP}}$ [g dry wt bacteria (mol ATP generated from catabolism) ${ }^{-1}$ ] values in comparison to ammonia as a nitrogen source. The production of polysaccharide with glutamine but not with 5oxoproline may reflect the utilization of all the glutamine supplied but only a fraction of the 5oxoproline. The exact mechanism by which glutamine stimulates polysaccharide formation is unknown.

In continous culture studies there will be little free ammonia present if glutamine or 5-oxoproline and ammonia are the growth limiting nutrients, nevertheless careful attention should be paid to the sterilization of glutamine solutions to avoid the production of ammonia and 5-oxoproline. The production of ammonia on heating aqueous glutamine solutions or the production of extracellular ammonia from filter-sterilized glutamine solutions by $R$. leguminosarum in batch culture (Ratcliffe et al., 1978) could complicate studies on the regulation of nitrogen fixation.

H.D. R. is grateful to the Science Research Council for a CASE Research Studentship. We would like to thank Mr L. J. Barnes for his technical aid and Mrs K. Shaw of Shell Research Ltd, Sittingbourne for C, H and N analysis.

\section{REFERENCES}

ARChibald, R. M. (1945). Chemical characteristics and physiological roles of glutamine. Chemical Reviews 37, 161-208.

Bergersen, F. J., Turner, G. L., Gibson, A. H. \& DUdMAN, W. F. (1976). Nitrogenase activity and respiration of cultures of Rhizobium spp. with special reference to the concentration of dissolved oxygen. Biochimica et biophysica acta 444, 164-174.

JONES, C. W. (1978). Microbial oxidative phosphorylation. Biochemical Society Transactions 6, 361363.
Jones, C. W., Brice, J. M. \& Edwards, C. (1977). The effect of respiratory chain composition on the growth efficiencies of aerobic bacteria. Archives of Microbiology 115, 85-93.

Kawai, J., KawaI, Y. \& Uemura, T. (1965). Studies on metabolism of pyrollidone carboxylic acid in bacteria. I. Accumulation of L-glutamic acid by bacteria in the presence of pyrollidone carboxylic acid. Agricultural and Biological Chemistry 29, 395-402.

KawaI, Y. \& Uemura, T. (1966). Studies on metabolism of pyrollidone carboxylic acid in bacteria. II. L- 
Glutamic acid formation from pyrollidone carboxylic acid by Pseudomonas alcaligenes. Agricultural and Biological Chemistry 33, 212-219.

KawaI, Y., Aida, K. \& Uemura, T. (1969). Studies on metabolism of pyrollidone carboxylic acid in bacteria. III. Cleavage of pyrollidone carboxylic acid to Lglutamic acid by the enzyme preparation obtained from Pseudomonas alcaligenes ATCC-12815, with special reference to the optical isomerisation of pyrollidone carboxylic acid by the enzyme preparation. Agricultural and Biological Chemistry 33, 212219.

MaruYama, Y. \& Nomura, M. (1956). Metabolism of pyrollidone carboxylic acid by bacteria. Journal of Biochemistry 43, 327-335.

RatClifFe, H. D., Drozd, J. W. \& Bull, A. T. (1978). The utilisation of L-glutamine and the product of its thermal decomposition by Klebsiella pneumoniae and Rhizobium leguminosarum. FEMS Microbiology Letters 3, 65-69.

RATClifFe, H. D., Drozd, J. W. \& Bull, A. T. (1983). Growth energetics of Rhizobium leguminosarum grown in chemostat culture. Journal of General Microbiology 129, 1697-1706.

Tosa, T. \& Chibata, I. (1965). Utilisation of cyclic amides and formation of $\omega$-amino acids by microorganisms. Journal of Bacteriology 89, 919-920.

VAN DER WERF, P. \& MEISTER, A. (1974). Isolation of 5-oxoprolinase from a prokaryote. Biochemical and Biophysical Research Communications 56, 90-96.

VAN DER Werf, P. \& MeISTer, A. (1975). The metabolic formation and utilisation of 5-oxo-L-proline ( $\mathrm{L}$ pyroglutamate, L-pyrrolidone carboxylate). $A d$ vances in Enzymology 43, 519-556.

Wilcockson, J. \& WerNer, D. (1978). Nitrogenase activity of Rhizobium japonicum growing on agar surfaces in relation to slime production, growth and survival. Journal of General Microbiology 108, 151160.

WILCOCKSON, J. \& WERnER, D. (1979). Organic acids and prolonged nitrogenase activity by non-growing, free-living Rhizobium japonicum. Archives of Microbiology 122, 153-159. 